\title{
Fuzzy Soft Set Multi-Attribute Decision Making Method Based on TOPSIS with Improved Entropy Weight
}

\author{
Yangfan Liu ${ }^{\text {a)}}$, Xiaohua Liu ${ }^{\text {b) }}$ \\ School of Computer and Community Lanzhou University of Technology Lanzhou Gansu 730050, China. \\ a) 592268386@qq.com \\ b)171422451@qq.com
}

\begin{abstract}
In the process of fuzzy soft set multi-attribute decision making, and It is difficult to determine the weight of the attribute parameters which results in low efficiency of decision making. Therefore, a new method of determining the entropy weight which is calculated by the notion of the importance degree and the deviation degree based on parameters, moreover entropy weight is used to determine the objective weight of attribute parameters. The dynamic weight determination method is proposed to determine the combined weight which combines the subjective weight and the objective weight, then combined with the traditional TOPSIS method, this paper proposes the method of fuzzy soft set multi-attribute decision making based on TOPSIS with improved entropy weight. Finally, a practical example has been analyzed to verify the feasibility and effectiveness of the approach, which can realize the dynamic decision making according to the demand of decision making and improve the efficiency of decision making.
\end{abstract}

Key words: Combined Weight; Entropy Weight; TOPSIS; Fuzzy Soft Set; Multi-attribute Decision Making.

\section{INTRODUCTION}

In real life, many problems often contain uncertainty and vagueness, which are difficult to describe and solve with traditional mathematical tools. In order to study these uncertainties, many scholars have put forward the mathematical theory to deal with the problem of uncertainty. At present, the mathematical theories dealing with the problem of uncertainty are: fuzzy set theory [1], rough set theory [2], interval number theory [3], probability theory, etc. However, these theories have the problem of insufficient parameterization tools. Thus, the Russian mathematician Molodstov [4] put forward the theory of soft sets, and the parameters of soft concentration are unconstrained, which is a new theory of dealing with uncertainties. Based on the study of soft set theory, many scholars put forward some new theories. Feng [5] and others put forward the concept of rough set, making soft set theory applied to rough set.Maji[6] and others combine fuzzy sets with soft sets theory to give the concept of fuzzy soft sets. Now fuzzy set has been widely used in various fields, such as economics, medicine and environmental science. Both in theoretical research and application fields have made great progress, especially its application in decision-making has been widely concerned.

In recent years, some achievements have been made in the research on decision-making about fuzzy soft sets. For example, Maji et al. [7] firstly combined soft-set theory with decision-making, and proposed a decision-making method based on soft-set theory. In literature [8], Feng et al. used horizontal soft sets to transform fuzzy soft sets into soft sets, and then made decisions based on the soft set. In the literature [9], a new parameter reduction algorithm based on parameter importance is proposed by using discernibility matrix to define the importance of parameters. Literature [10] proposed the fuzzy soft sets of soft information sequence relationship, the use of soft information ordinal fuzzy set decision-making. Literature [11] combines the relationship of the dominance of interval values with the probability and defines the average degree of comprehensive dominance and proposes an improved ranking method of the dominance relation. Literature [12] gives the parameter weight determination method and proposes a 
multi-person decision algorithm based on weighted fuzzy sets. Although the methods mentioned above have some advantages in the decision-making of fuzzy soft sets, they do not make full use of the unconstrained features of fuzzy soft sets. They often neglect the influence of the weights of parameters on the decision-making results, Resulting in excessive calculation of decision-making, unreasonable decision-making results and low decision-making efficiency.

In the process of fuzzy soft multi-attribute decision-making, the weight information of attribute parameters plays an important role in the final decision-making. How to effectively determine the weight of the parameters is a hot research topic in fuzzy soft-set multi-attribute decision making. Entropy weight method can effectively determine the objective weight, but the traditional dispersion degree is used to determine the entropy power, simply use parameters to measure the distance between the importance of the parameter is ignored itself to determine the effect of entropy weight. At the same time, most of the fuzzy sets of soft multi-attribute decision-making can only make a single decision based on objective data to get the only decision result and cannot make full use of the decision data provided to achieve dynamic decision-making, so that the decision-making efficiency is greatly reduced and the decisionmaking result is unreasonable. Based on the above insufficiency, this paper uses the new entropy weight determination method to calculate the entropy weight by defining the importance and degree of the parameters, using the entropy weight to determine the objective weights, and applying the TOPSIS method to the fuzzy soft set multi-attribute decision-making. We use the parameter importance to improve the entropy to determine the objective weight and give the method to determine the dynamic weight. Combining the subjective weight with the objective weight, we get the combined weight. Combined with the traditional TOPSIS method, we propose the TOPSIS fuzzy soft set Attribute decision-making method. Make the decision-making method more flexible and make the decision-making result more reasonable.

\section{BASIC CONCEPT}

The concept of "Entropy" stemmed from thermodynamics, which was introduced by Clausius, a German physicist, in 1850 to measure the degree of disorder in the system. Information entropy is a generalized concept of entropy. Shannon, the father of information theory, introduced it into information theory in 1948 to measure the uncertainty of signal in the source. Entropy weight actually describes the degree of disorder of the system, which is a measure of the uncertainty of the information and can measure the amount of information it contains. In the fuzzy soft-set multiattribute decision-making, the more information the attribute parameter contains, the greater the effect of the attribute parameter on the decision.Assuming there are ${ }^{m}$ to be decision objects, each decision object has ${ }^{n}$ attribute parameters,

$x_{i j}$ is the value of the ${ }^{j}$ th attribute parameter of the ${ }^{i}$ th decision object, then the entropy weight of the ${ }^{j}$ th attribute parameter is:

$$
e_{j}=-\frac{1}{\ln m} \sum_{i=1}^{m} p_{i j} \ln p_{i j}
$$

among them, $p_{i j}=x_{i j} / \sum_{i=1}^{m} x_{i j}$, That is, $p_{i j}$ represents the proportion of the $i$ th decision object in the attribute parameter under the $j$ th attribute parameter. Generally, the weight determined by the entropy method is more objective and credible.

TOPSIS (Technique for Order Preference by Similarity to Ideal Solution) was first proposed by C.L. Hwang and K.Yoon in 1981.The basic idea is to establish an initial decision matrix first, and then find out the optimal solution and the worst solution in the limited solution based on the normalized initial matrix, that is, positive and negative ideal solutions, and then calculate each evaluation object and the optimal solution The distance from the worst case approach leads to a solution that is as close to positive as possible, while keeping as far as possible from negative ones[14].The method is reasonable and easy to understand, and the calculation is relatively simple. The method can be used to describe the performance of alternative solutions in a simple mathematical form and objective weights can be used in the comparison process [15].

The following fuzzy set of knowledge related to a brief introduction:

Definition 1[4] Set $U$ is a finite non-empty domain, $E$ is a set of parameters, $P(U)$ is a power set of domain $U$, and $A \subseteq E$.I'm going to call the order $(F, A)$ for the soft set on the domain $U$, where $F$ is the mapping, $F: A \rightarrow P(U)$. The soft set of domain $U$ can be regarded as a parameter family composed of a subset of $U$. Therefore, for each $e \in A, F(E)$ can be considered as the $e$-approximate set of the $\operatorname{soft} \operatorname{set}(F, A)$. 
Definition 2[6] Set $(\tilde{F}, A)$ is the fuzzy soft set on the domain $U, A \subseteq E$ and $E$ is the parameter set. Then $\tilde{F}$ is a function of $U \times A$ to $V$, which is $\tilde{F}: U \times A \rightarrow V, \tilde{F}\left(e_{j}\right)(x) \in V=[0,1]$, where $x \in U, e_{j} \in A, V=[0,1], \tilde{F}\left(e_{j}\right)(x)$ is the membership of object $x$ on parameter $e_{j}$.

Example 1 Suppose the fuzzy set $(\tilde{F}, A)$ describes the "characteristics of Mr. X's purchase of a car", domain $U=\left\{x_{1}, x_{2}, x_{3}, x_{4}, x_{5}, x_{6}\right\}$ represents the set of 6 optional vehicles, the parameter set $E=\left\{e_{1}, e_{2}, e_{3}, e_{4}\right\}$ represents the characteristics of the car, where $e_{1}$ represents "brand", $e_{2}$ represents "configuration", $e_{3}$ On behalf of "performance", $e_{4}$ on behalf of "after sales." If $\tilde{F}\left(e_{1}\right)=\left\{\frac{x_{1}}{0.7}, \frac{x_{2}}{0.8}, \frac{x_{3}}{0.9}, \frac{x_{4}}{0.3}, \frac{x_{5}}{0.5}\right\}, \tilde{F}\left(e_{2}\right)=\left\{\frac{x_{1}}{0.9}, \frac{x_{2}}{0.2}, \frac{x_{3}}{0.2}, \frac{x_{4}}{0.7}, \frac{x_{5}}{0.8}\right\}, \tilde{F}\left(e_{3}\right)=\left\{\frac{x_{1}}{0.4}, \frac{x_{2}}{0.7}, \frac{x_{3}}{0.2}, \frac{x_{4}}{0.6}, \frac{x_{5}}{0.1}\right\}$, $\tilde{F}\left(e_{4}\right)=\left\{\frac{x_{1}}{0.6}, \frac{x_{2}}{0}, \frac{x_{3}}{0.8}, \frac{x_{4}}{0.6}, \frac{x_{5}}{0.5}\right\}$.In the form of a fuzzy soft set, it's $(\tilde{F}, A)=\left\{\tilde{F}\left(\mathrm{e}_{1}\right), \tilde{F}\left(\mathrm{e}_{2}\right), \tilde{F}\left(\mathrm{e}_{3}\right), \tilde{F}\left(\mathrm{e}_{4}\right)\right\}$.

Fuzzy soft set from the Angle of the parameterized description of objective things, and cannot accurately describe the thing itself, is not good or bad, but the description of the "good" or "bad" in the middle of the state, has a certain ambiguity. At the same time, the fuzzy soft set can be expressed in the form of two-dimensional table, as shown in table 1:

TABLE 1. Fuzzy $\operatorname{soft} \operatorname{set}(\tilde{F}, A)$

\begin{tabular}{ccccc}
\hline$U$ & $e_{1}$ & $e_{2}$ & $e_{3}$ & $e_{4}$ \\
\hline$x_{1}$ & 0.7 & 0.9 & 0.4 & 0.6 \\
$x_{2}$ & 0.8 & 0.2 & 0.7 & 0 \\
$x_{3}$ & 0.9 & 0.2 & 0.2 & 0.8 \\
$x_{4}$ & 0.3 & 0.7 & 0.6 & 0.6 \\
$x_{5}$ & 0.5 & 0.8 & 0.1 & 0.5 \\
\hline
\end{tabular}

THE IMPROVED ENTROPY WEIGHT METHOD DETERMINES THE PARAMETER WEIGHT

In the fuzzy soft-set multi-attribute decision-making, the weight of the size of a direct impact on the final decisionmaking results. Therefore, the weight determination becomes an important research topic.

\section{The Importance of Parameters}

Definition 3 Set $(\tilde{F}, A)$ is the fuzzy soft set on the domain $U$, and $E$ is the parameter set. For any parameter set $A \subseteq E, R_{A}^{2}$ is the dominant relation of fuzzy soft set. Namely $R_{A}^{2}=\left\{\left(x_{m}, x_{n}\right) \in U \times U \mid \tilde{F}\left(e_{j}\right)\left(x_{m}\right) \geq \tilde{F}\left(e_{j}\right)\left(x_{n}\right), \forall e_{j} \in A\right\}$.

If $\tilde{F}\left(e_{j}\right)\left(x_{m}\right) \geq \tilde{F}\left(e_{j}\right)\left(x_{n}\right)$, under parameter $e_{j}$, object $x_{m}$ is more advantageous than object $x_{n}$ in fuzzy soft set multiattribute decision making, which is more important to the effect of decision results.

Definition 4 Set $(\tilde{F}, A)$ is the fuzzy soft set on the domain $U, \forall x_{m}, x_{n} \in U, A \subseteq E$, definition: $d\left(x_{m}, x_{n}\right)=\left\{\mathrm{e}_{j}: \tilde{\mathrm{F}}\left(\mathrm{e}_{j}\right)\left(x_{m}\right) \geq \tilde{\mathrm{F}}\left(\mathrm{e}_{j}\right)\left(x_{n}\right)\right\}$ is the dominant parameter set of $x_{m}$ and $x_{n}$, and the matrix $D=\left(d\left(x_{m}, x_{n}\right)\right)_{m, n \leq|U|}$ is the dominant matrix. Among them, the dominant matrix is $|U| \times|U|$ dimensional matrix, $m \neq n$ and the diagonal of the dominant matrix is empty set.

By definition 4 can have the following properties:

(1) $0 \leq d\left(x_{m}, x_{n}\right) \leq|E|, d\left(x_{m}, x_{m}\right)=E$

(2) $d\left(x_{m}, x_{n}\right)=E, x_{m} \in\left[x_{n}\right]_{E}^{2}$

(3) $|E| \leq\left|d\left(x_{m}, x_{n}\right)\right|+\left|d\left(x_{n}, x_{m}\right)\right|$

Proof :(1)By definition 4, we can get: when satisfied $0 \leq d\left(x_{m}, x_{n}\right) \leq|E|, d\left(x_{m}, x_{m}\right)=\left\{\mathrm{e}_{j}: \tilde{\mathrm{F}}\left(\mathrm{e}_{j}\right)\left(x_{m}\right) \geq \tilde{\mathrm{F}}\left(\mathrm{e}_{j}\right)\left(x_{m}\right)\right\}$,in the dominant matrix, for object $x_{m}$, there is only one condition for the condition to satisfy $\tilde{\mathrm{F}}\left(\mathrm{e}_{j}\right)\left(x_{m}\right) \geq \tilde{\mathrm{F}}\left(\mathrm{e}_{j}\right)\left(x_{m}\right)$, that is $\tilde{\mathrm{F}}\left(\mathrm{e}_{j}\right)\left(x_{m}\right)=\tilde{\mathrm{F}}\left(\mathrm{e}_{j}\right)\left(x_{m}\right)$, there is no case of $\tilde{\mathrm{F}}\left(\mathrm{e}_{j}\right)\left(x_{m}\right)>\tilde{\mathrm{F}}\left(\mathrm{e}_{j}\right)\left(x_{m}\right)$, at this point $d\left(x_{m}, x_{m}\right)=\left\{e_{j}: \tilde{F}\left(e_{j}\right)\left(x_{m}\right)=\tilde{F}\left(e_{j}\right)\left(x_{m}\right)\right\}$, that is $d\left(x_{m}, x_{m}\right)=E$. 
(2)When $d\left(x_{m}, x_{n}\right)=E,\left\{e_{j}: \tilde{F}\left(e_{j}\right)\left(x_{m}\right) \geq \tilde{F}\left(e_{j}\right)\left(x_{n}\right)\right\}=E$, at this point, for parameter set $E$, all parameters are satisfied with the condition $\tilde{F}\left(e_{j}\right)\left(x_{m}\right) \geq \tilde{F}\left(e_{j}\right)\left(x_{n}\right)$, and the object $x_{m}$ is the dominant class of object $x_{n}$ in the parameter set $E$, namely $x_{m} \in\left[x_{n}\right]_{E}^{2}$.

(3)In the fuzzy soft set $(\tilde{F}, A)$, let $\left|\left\{e_{j}: \tilde{F}\left(e_{j}\right)\left(x_{m}\right)>\tilde{F}\left(e_{j}\right)\left(x_{n}\right)\right\}\right|=p,\left|\left\{e_{j}: \tilde{F}\left(e_{j}\right)\left(x_{n}\right)>\tilde{F}\left(e_{j}\right)\left(x_{m}\right)\right\}\right|=q\left|\left\{e_{j}: \tilde{F}\left(e_{j}\right)\left(x_{m}\right)=\tilde{F}\left(e_{j}\right)\left(x_{n}\right)\right\}\right|=r$, we can get $p+q+r=|E|$, and the parameter set $E$ satisfies this relationship, and $d\left(x_{m}, x_{n}\right)=\left\{e_{j}: \tilde{F}\left(e_{j}\right)\left(x_{m}\right)>\tilde{F}\left(e_{j}\right)\left(x_{n}\right)\right.$ or $\left.\tilde{F}\left(e_{j}\right)\left(x_{m}\right)=\tilde{F}\left(e_{j}\right)\left(x_{n}\right)\right\}$, we can get: 2

$\left|d\left(x_{m}, x_{n}\right)\right|=\mid\left\{e_{j}: \tilde{F}\left(e_{j}\right)\left(x_{m}\right)>\tilde{F}\left(e_{j}\right)\left(x_{n}\right\}|+| e_{j}: \tilde{F}\left(e_{j}\right)\left(x_{m}\right)=\tilde{F}\left(e_{j}\right)\left(x_{n}\right\} \mid\right.$, that is, $\left|d\left(x_{m}, x_{n}\right)\right|=p+r$; That's the same thing as $\left|d\left(x_{n}, x_{m}\right)\right|=q+r$.

Therefore, $\left|d\left(x_{m}, x_{n}\right)\right|+\left|d\left(x_{n}, x_{m}\right)\right|=(p+r)+(q+r)$,it can be further obtained: $\left|d\left(x_{m}, x_{n}\right)\right|+\left|d\left(x_{n}, x_{m}\right)\right|=p+q+2 r$. and $p+q+r=|E|$, so when $r=0,\left|d\left(x_{m}, x_{n}\right)\right|+\left|d\left(x_{n}, x_{m}\right)\right|=|E|$; When $r \neq 0$, at this time $r$ is positive integer, can get $p+q+2 r>p+q+r$, namely $\left|d\left(x_{m}, x_{n}\right)\right|+\left|d\left(x_{n}, x_{m}\right)\right|>E$.

To sum up $E \leq\left|d\left(x_{m}, x_{n}\right)\right|+\left|d\left(x_{n}, x_{m}\right)\right|$.

In the dominance matrix, each parameter represents the dominant attribute of the object and contributes to determining the dominance of the object, meaning that these attributes are important for determining the dominance relationship, but of different significance. The higher the frequency of an attribute in the dominant matrix, the greater the proportion of the attribute, the more identifiable dominant nodes the attribute will have. This is advantageous for determining the dominant relationship in the dominant matrix, and the corresponding attribute is advantageous the greater the importance of the matrix; the other hand, the less important.

Definition 5 Set $(\tilde{F}, E)$ is the fuzzy soft set on the domain $U, D=\left(d\left(x_{m}, x_{n}\right)\right)$, and $m, n \leq|U|$ is the advantage matrix of fuzzy soft set. Among them, $d\left(x_{m}, x_{n}\right)$ is the element of $m$ row $n$ column in the advantage matrix $D=\left(d\left(x_{m}, x_{n}\right)\right)$ of fuzzy soft set $(\tilde{F}, E)$. For $\forall e_{j} \in E$, the frequency of parameter $e_{j}$ can be expressed as: $d\left(e_{j}\right)=\sum_{m, n=1,2, \cdots U \mid}\left|e_{j} \cap d\left(x_{m}, x_{n}\right)\right|$, the frequency is normalized, and the importance of parameter $e_{j}$ is as follows:

$$
\alpha\left(e_{j}\right)=d\left(e_{j}\right) / \sum_{j=1}^{|E|} d\left(e_{j}\right)
$$

If $(\tilde{F}, E)$ is a fuzzy soft set on the domain $U$, the importance of parameter $e_{j}$ on the advantage matrix $D=\left(d\left(x_{m}, x_{n}\right)\right)_{m, n \leq U \mid}$ satisfies the following properties:

(1) Boundedness: $0 \leq \alpha\left(e_{j}\right) \leq 1$

(2) $\sum_{j=1,2, \cdots, E \mid} \alpha\left(e_{j}\right)=1$

(3) Transitivity: if $\alpha\left(e_{i}\right) \geq \alpha\left(e_{j}\right), \alpha\left(e_{j}\right) \geq \alpha\left(e_{k}\right)$, then $\alpha\left(e_{i}\right) \geq \alpha\left(e_{k}\right)$

Proof:(1) By definition 4, the property (1) can be obtained $0 \leq d\left(x_{m}, x_{n}\right) \leq|E|,\left|e_{j} \cap d\left(x_{m}, x_{n}\right)\right|=\{0,1\}$, when $\left|e_{j} \cap d\left(x_{m}, x_{n}\right)\right|=0, d\left(e_{j}\right)$ has the minimum value $d\left(e_{j}\right)_{\min }=0$; When $\left|e_{j} \cap d\left(x_{m}, x_{n}\right)\right|=1, d\left(e_{j}\right)$ has a maximum $d\left(e_{j}\right)_{\max }=|U|^{2}$, so $0 \leq d\left(e_{j}\right) \leq|U|^{2}$, and $d\left(e_{j}\right)$ is a bounded non-negative integer, and $\alpha\left(e_{j}\right)=d\left(e_{j}\right) / \sum_{j=1}^{|E|} d\left(e_{j}\right)$,so $0 \leq \alpha\left(e_{j}\right) \leq 1$ is true.

(2) $\sum_{j=1,2, \cdots, E \mid} \alpha\left(e_{j}\right)=\sum_{j=1,2, \cdots, E \mid}\left(d\left(e_{j}\right) / \sum_{j=1}^{|E|} d\left(e_{j}\right)\right)$

$=\sum_{j=1}^{|E|} d\left(e_{j}\right) / \sum_{j=1}^{|E|} d\left(e_{j}\right)=1$

(3) Transmissibility is directly verifiable by definition 5 .

Theorem 1 Set $(\tilde{F}, E)$ is the fuzzy soft set on the domain $U, E$ is the parameter set, and if the importance of the parameter satisfies $\alpha\left(e_{i}\right)>\alpha\left(e_{j}\right)$, parameter $e_{i}$ is more important than parameter $e_{j}$ in fuzzy soft set multi-attribute decision making. 
Proof :Because when the parameter is important enough to satisfy $\alpha\left(e_{i}\right)>\alpha\left(e_{j}\right), d\left(e_{i}\right) / \sum_{i=1}^{|E|} d\left(e_{i}\right)>d\left(e_{j}\right) / \sum_{j=1}^{|E|} d\left(e_{j}\right)$ can be obtained, which is further available to $\sum_{i=1}^{|E|} d\left(e_{i}\right)=\sum_{j=1}^{|E|} d\left(e_{j}\right)$, and $d\left(e_{i}\right)>d\left(e_{j}\right) \quad$,namely $\sum_{m, n=1,2, \cdots, U \mid}\left|e_{i} \cap d\left(x_{m}, x_{n}\right)\right|>\sum_{m, n=1,2, \cdots, \cdots \mid}\left|e_{j} \cap d\left(x_{m}, x_{n}\right)\right|$. Therefore, in the dominant matrix, the parameter $e_{i}$ has more frequency than the parameter $e_{j}$, and the corresponding parameter occupies a larger proportion in the dominant matrix, which is more advantageous for determining the dominant relationship of the parameters in the dominant matrix. Therefore, the more important the fuzzy soft set multi-attribute decision-making effect is. So, when $\alpha\left(e_{i}\right)>\alpha\left(e_{j}\right)$, parameter $e_{i}$ is more important than parameter $e_{j}$ in fuzzy soft-set multi-attribute decision making.

\section{The Deviation of the Parameter}

In fuzzy soft concentration, according to the principle of information entropy, if the difference between attribute parameter values is larger, the data between attribute parameters will be more scattered, the greater the parameter uncertainty will be, and the more effective information will be carried, the more important is the role of the outcome of the decision. That is, the more dispersed the attribute parameter value, the more weight value should be given. Therefore, we measure the degree of dispersion by measuring the degree of dispersion of the data to measure the magnitude of the weights.

Definition 6 Set $(\tilde{F}, E)$ is the fuzzy soft set on the domain $U, \forall x_{i}, x_{j} \in U, \forall e_{j} \in E, u_{i j}$ is the parameter value of the domain object $x_{i}$ in parameter $e_{j}$, namely $\left\{\mathrm{u}_{i j} \mid \mathrm{u}_{i j}=F\left(e_{j}\right)\left(x_{i}\right), i=1,2 \cdots, m, j=1,2, \cdots, n\right\}$. For parameter $e_{j}$, the dispersion between object $x_{i}$ and other objects is:

$$
D_{i j}=\sum_{k=1}^{m} D\left(u_{i j}, u_{k j}\right)=\sqrt{\sum_{k=1}^{m}\left(u_{i j}-u_{k j}\right)^{2}}, i \in 1,2 \cdots m, j \in 1,2 \cdots n
$$

The total dispersion degree of the fuzzy soft concentrated parameter $e_{j}$ in the object is:

$$
D_{j}=\sum_{i=1}^{m} D_{i j}=\sum_{i=1}^{m} \sqrt{\sum_{k=1}^{m}\left(u_{i j}-u_{k j}\right)^{2}}, \quad i \in 1,2, \cdots, m
$$

The deviation degree has the following properties:

(1) Boundedness: $0 \leq D_{j} \leq m^{2}$

(2) If $D_{i}>D_{j}$, parameter $e_{i}$ is more dispersed than parameter $e_{j}$.

(3) Transitivity: if $D_{i}>D_{j}, D_{j}>D_{k}$, then $D_{i}>D_{k}$

Proof:(1) Because $u_{i j}$ is the parameter value of the fuzzy soft set, namely $0 \leq u_{i j} \leq 1$, so $0 \leq \sqrt{\left(u_{i j}-u_{k j}\right)^{2}} \leq 1$, $0 \leq \sqrt{\sum_{k=1}^{m}\left(u_{i j}-u_{k j}\right)^{2}} \leq m$, namely $0 \leq D_{i j} \leq m$. And $D_{j}=\sum_{i=1}^{m} D_{i j}$, so the condition $0 \leq D_{j} \leq m^{2}$ is set up.

(2) When $D_{i}>D_{j}$, by definition 6, $\sum_{a=1}^{m} D_{a i}>\sum_{a=1}^{m} D_{a j} \quad, \quad \sum_{a=1}^{m} \sum_{k=1}^{m} D\left(u_{a i}-u_{k i}\right)>\sum_{a=1}^{m} \sum_{k=1}^{m} D\left(u_{a j}-u_{k j}\right) \quad$,namely $\sum_{a=1}^{m} \sum_{k=1}^{m} D\left(u_{a i}-u_{k i}\right)>\sum_{a=1}^{m} \sum_{k=1}^{m} D\left(u_{a j}-u_{k j}\right)$. As a result, it can be obtained that the difference between the parameter value and the distance difference of each item in parameter $e_{i}$ is larger than that in parameter $e_{j}$, and the difference between the parameter value and other parameters is more obvious. Therefore, when the parameter's deviation degree is $D_{i}>D_{j}$, the property parameter $e_{i}$ is greater than the dispersion degree of parameter $e_{j}$.

(3) Transmission is defined by definition 6. 


\section{Improved Entropy Weight Method}

The entropy in information theory is used to measure the degree of disorder of the system. It can also be used to measure the amount of valid information carried by data. Therefore, the entropy of information can be used to determine the weight information. The more dispersed the data, the greater the degree of uncertainty of the information. The degree of uncertainty can be measured by the degree of dispersion. At the same time, the greater the importance of parameters, the more information they carry, and the greater the effect of this parameter on decision-making. However, the traditional entropy method usually only considers the influence of the data dispersion size on the weight but neglects the influence of the relative importance of the parameter itself on the determination of dispersion. Aiming at the above problems, this paper proposes a new method to calculate entropy, which combines the degree of dispersion and importance to determine the information of entropy, which avoids considering only the degree of data dispersion when calculating entropy and neglects the relative importance of the parameter itself The Effect of Degree on Determining Entropy. This improved method makes the certain entropy more reasonable and scientific.

The improved entropy weight method calculates the objective weight information process of attribute parameters as follows:

(1) Calculate the entropy weight according to the parameter deviation degree and importance degree:

$$
E_{j}=-k \sum_{i=1}^{m} \alpha\left(e_{j}\right) \frac{D_{i j}}{D_{j}} \ln \alpha\left(e_{j}\right) \frac{D_{i j}}{D_{j}}, \quad i=1,2, \cdots m, j=1,2, \cdots n
$$

Among them: The coefficient $k=\frac{1}{\ln m}$ is a constant, provides when $\alpha\left(e_{j}\right) \frac{D_{i j}}{D_{j}}=0, \alpha\left(e_{j}\right) \frac{D_{i j}}{D_{j}} \ln \alpha\left(e_{j}\right) \frac{D_{i j}}{D_{j}}=0$.

(2) Calculate the difference coefficient $d_{j}$ of the attribute parameter $e_{j}$ :

$$
d_{j}=1-E_{j}, \quad j=1,2, \cdots n
$$

(3) Calculate objective weight information $\omega_{j}$ :

$$
\omega_{j}=\frac{d_{j}}{\sum_{i=1}^{n} d_{j}}, j=1,2, \cdots n
$$

This improved entropy method determines the objective weight, not only considering the influence of the degree of dispersion between objects on entropy under the same parameter, but also considering the influence of the relative importance between parameters on the objective weight, combining the degree of dispersion and importance, so that the weight information obtained is more comprehensive and the calculation result is more accurate.

\section{Determination of the Combined Weight}

The weight of the parameter includes the subjective weight and the objective weight. The subjective weight is given by the decision makers according to their own experience, knowledge level and personal preferences and other information parameters of the weight, the subjective weight is strong, the calculation process is relatively simple; the objective weight Strict dependence on objective data, according to certain rules and rules to determine the weight of the attribute parameters. Objective weight does not depend on the subjective experience of decision-makers, weight information has better accuracy, but the theory is strong, the calculation process may be more complicated. In the case of fuzzy soft-set multi-attribute decision-making, if only a single weighting information is used for decision-making, the decision-making results tend to be subjective or bias-oriented, which is not in accordance with the actual situation. In this paper, we consider the influence of subjective weight and objective weight, in which the subjective weight is given by the expert, the objective weight is determined by the improved entropy method, the method of determining the dynamic weight is given, and the subjective weight and the objective weight are combined to obtain the combined weight.

In the process of fuzzy soft-set multi-attribute decision-making, in order to make the decision-making result more in line with the actual decision-making requirements, we need to get reasonable weight information. Suppose that 
$\beta=\left(\beta_{1}, \beta_{2}, \cdots, \beta_{n}\right)$ is the subjective weight given by the experts according to their own experience, and $\omega=\left(\omega_{1}, \omega_{2}, \cdots, \omega_{n}\right)$ is the objective weight obtained by the improved entropy weight method, so that the combination weight is $\lambda=\left(\lambda_{1}, \lambda_{2}, \cdots, \lambda_{n}\right)$, and if $\lambda_{j}$ meets the following conditions:

$$
\left\{\begin{array}{l}
\lambda_{j}=\theta \beta_{j}+(1-\theta) \omega_{j} \\
0 \leq \theta \leq 1
\end{array}\right.
$$

Among them, $\theta$ is the dynamic adjustment coefficient, which is used to adjust the proportion of subjective weight and objective weight. It can be seen from formula (8) that the size of the combined weight $\lambda_{j}$ changes with the change of $\theta$. When $\theta=0$, the combined weight is completely changed to objective weight; When $\theta=1$, the combined weight is completely subjective. At the same time, the decision maker can adjust the combination weight coefficient artificially according to different decision demands to meet different decision needs and realize the purpose of dynamic decision-making. This combination of weights can make the decision more flexible and scientific, more in line with the requirements of fuzzy soft-set multi-attribute decision-making.

\section{TOPSIS FUZZY SOFT DECISION METHOD BASED ON IMPROVED ENTROPY}

The basic idea of TOPSIS fuzzy soft sets multi-attribute decision-making method to improve entropy is as follows: Firstly, the objective weights are determined by the improved entropy method, then combined with the subjective weights to form the combined weights, and the combined weights can be adjusted by the dynamic adjustment coefficients Weight ratio, in order to meet the needs of dynamic decision-making. Then, weighted fuzzy sets are obtained by using the combined weights. Finally, the optimal decision objects of fuzzy soft sets are obtained by combining with the traditional TOPSIS method.

Set $(\tilde{F}, E)$ is the fuzzy soft set of $U$ in the domain, $X=\left\{x_{1}, x_{2}, \cdots, x_{m}\right\}$ is the set of alternative decision objects for fuzzy soft set, and $E=\left\{e_{1}, e_{2}, \cdots, e_{n}\right\}$ is the set of attribute parameters of fuzzy soft set.

Decision-making procedure of fuzzy soft-set multi-attribute decision making method based on improved entropy TOPSIS:

Input: fuzzy soft set $S=(\tilde{F}, E)$

Output: the optimal decision object of fuzzy soft set

Step1: Constructing decision matrix $P=\left(u_{i j}\right)_{m \times n}$ of fuzzy soft sets according to decision conditions.

Step2: The combined weight $\lambda_{j}$ of fuzzy soft set attribute parameters is determined according to the decision matrix $P$.

Step3: Calculation Weighted Evaluation Fuzzy Sets $Q$.

Weighted Evaluation Fuzzy Sets $Q=\left(v_{i j}\right)_{m \times n}=\left(\lambda_{j} \times u_{i j}\right)_{m \times n}, i=1,2, \cdots, m, j=1,2, \cdots, n$.

Step4: Determine the positive and negative ideal solutions of weighted fuzzy sets $Q$.

Positive ideal solution: $v^{+}=\left(v_{1}^{+}, v_{2}^{+}, \cdots, v_{j}^{+}\right)$, among them $v_{j}^{+}=\max _{i=1,2, \cdots, m}\left(v_{i j}\right), j=1,2, \cdots, n$.

Negative ideal solution: $v^{-}=\left(v_{1}^{-}, v_{2}^{-}, \cdots, v_{j}^{-}\right)$, among them $u_{j}^{-}=\min _{i=1,2, \cdots, m}\left(v_{i j}\right), j=1,2, \cdots, n$.

Step5: Calculate the distance between each decision object and the positive and negative ideal solutions and get the comprehensive evaluation index.

The distance between the decision object $x_{i}$ and the ideal solution of fuzzy soft set is:

$$
x_{i}^{+}=\sqrt{\sum_{j=1,2, \cdots, n}\left(v_{i j}-v_{j}^{+}\right)^{2}}
$$

The distance between the decision object $x_{i}$ and the negative ideal solution of fuzzy soft set is:

$$
x_{i}^{-}=\sqrt{\sum_{j=1,2, \cdots, n}\left(v_{i j}-v_{j}^{-}\right)^{2}}
$$

Calculate the comprehensive evaluation index for each decision object.

Comprehensive evaluation index $C_{i}$ of decision-making objects $x_{i}$ :

$$
C_{i}=\frac{x_{i}^{-}}{x_{i}^{-}+x_{i}^{+}}(i=1,2, \cdots, m)
$$

Step6: According to the comprehensive evaluation index, the fuzzy soft set decision objects are sorted and optimized. The larger the value of $C_{i}$, the better the corresponding decision objects. 


\section{THE EXAMPLE ANALYSIS}

Example2 A unit of civil servants for cadre selection, selected from five candidate section staff as section manager, there are four assessment indicators: work ability, personal qualities, cultural qualifications, leadership skills. The set of 5 candidates is $U=\left\{x_{1}, x_{2}, x_{3}, x_{4}, x_{5}\right\}$, The set of attribute parameters of the four assessment indicators is $E=\left\{e_{1}, e_{2}, e_{3}, e_{4}\right\}$, The subjective weight given by the expert group to the evaluation index parameters is $\beta_{j}=\{0.4,0.3,0.1,0.2\}$, The score values of each candidate in each assessment index are given in the form of fuzzy soft set:

$$
\tilde{F}\left(e_{1}\right)=\left\{\frac{x_{1}}{0.4}, \frac{x_{2}}{0.7}, \frac{x_{3}}{0.5}, \frac{x_{4}}{0.6}, \frac{x_{5}}{0.8}\right\}, \tilde{F}\left(e_{2}\right)=\left\{\frac{x_{1}}{0.8}, \frac{x_{2}}{0.6}, \frac{x_{3}}{0.7}, \frac{x_{4}}{0.6}, \frac{x_{5}}{0.3}\right\}, \tilde{F}\left(e_{3}\right)=\left\{\frac{x_{1}}{0.6}, \frac{x_{2}}{0.4}, \frac{x_{3}}{0.6}, \frac{x_{4}}{0.9}, \frac{x_{5}}{0.3}\right\}, \tilde{F}\left(e_{4}\right)=\left\{\frac{x_{1}}{0.5}, \frac{x_{2}}{0.4}, \frac{x_{3}}{0.5}, \frac{x_{4}}{0.4}, \frac{x_{5}}{0.8}\right\} .
$$

Based on the data provided, select the most suitable person from the five candidates as the section chief.

Step1: Building a fuzzy soft decision matrix $P$, as shown in Table 2:

TABLE 2. Decision matrix $P$ of fuzzy soft set

\begin{tabular}{ccccc}
\hline$P$ & $e_{1}$ & $e_{2}$ & $e_{3}$ & $e_{4}$ \\
\hline$x_{1}$ & 0.4 & 0.8 & 0.6 & 0.5 \\
$x_{2}$ & 0.7 & 0.6 & 0.4 & 0.4 \\
$x_{3}$ & 0.5 & 0.7 & 0.6 & 0.5 \\
$x_{4}$ & 0.6 & 0.6 & 0.9 & 0.4 \\
$x_{5}$ & 0.8 & 0.3 & 0.6 & 0.8 \\
\hline
\end{tabular}

Step2: Determine the combined weight $\lambda_{j}$ of fuzzy sets decision attributes:

(1) Calculate the importance of each parameter $\alpha\left(e_{j}\right)$.

According to Definition 4, we get the following matrix of advantages:

According to the formula (2), the importance of each parameter is:

$$
\left[\begin{array}{ccccc}
\phi & e_{2} e_{3} e_{4} & e_{2} e_{3} e_{4} & e_{2} e_{4} & e_{2} e_{3} \\
e_{1} & \phi & e_{1} & e_{2} e_{4} & e_{2} \\
e_{1} e_{3} e_{4} & e_{2} e_{3} e_{4} & \phi & e_{2} e_{4} & e_{2} e_{3} \\
e_{1} e_{3} & e_{2} e_{3} e_{4} & e_{1} e_{3} & \phi & e_{2} e_{3} \\
e_{1} e_{3} e_{4} & e_{1} e_{3} e_{4} & e_{1} e_{3} e_{4} & e_{1} e_{4} & \phi
\end{array}\right]
$$

$\alpha\left(e_{1}\right)=0.2000, \alpha\left(e_{2}\right)=0.2444, \alpha\left(e_{3}\right)=0.2889, \alpha\left(e_{4}\right)=0.2667$.

(2) Calculate the degree of dispersion of each parameter.

According to equation (3) (4) available dispersion $D_{i j}$, as shown in Table 3:

TABLE 3. Dispersion table

\begin{tabular}{ccccc}
\hline$D_{i j}$ & $e_{1}$ & $e_{2}$ & $e_{3}$ & $e_{4}$ \\
\hline$x_{1}$ & 0.5477 & 0.5831 & 0.3606 & 0.3317 \\
$x_{2}$ & 0.3873 & 0.3742 & 0.6083 & 0.4243 \\
$x_{3}$ & 0.3873 & 0.4359 & 0.3606 & 0.3317 \\
$x_{4}$ & 0.3162 & 0.3742 & 0.7211 & 0.4243 \\
$x_{5}$ & 0.5477 & 0.7681 & 0.3606 & 0.7071 \\
\hline$D_{j}$ & 2.1862 & 2.5355 & 2.4112 & 2.2191 \\
\hline
\end{tabular}

(3) Calculate the objective weight $\lambda_{j}$.

According to the importance and dispersion of the parameters, we can get the entropy $E_{j}$, the difference coefficient $d_{j}$, the objective weight $\omega_{j}$, as shown in Table 4: 
TABLE 4. Objective weight information table

\begin{tabular}{ccccc}
\hline & $e_{1}$ & $e_{2}$ & $e_{3}$ & $e_{4}$ \\
\hline$E_{j}$ & 0.3970 & 0.4520 & 0.5031 & 0.4785 \\
$d_{j}$ & 0.6030 & 0.5480 & 0.4969 & 0.5215 \\
$\omega_{j}$ & 0.2780 & 0.2526 & 0.2290 & 0.5215 \\
\hline
\end{tabular}

(4) Calculate the combined weight $\lambda_{j}$.

It is known that the subjective weight given by people is $\beta_{j}=\{0.4,0.3,0.1,0.2\}$, and the objective weight is $\omega_{j}=\{0.2780,0.2526,0.2161,0.2364\}$. According to formula (9), the weight can be combined: $\lambda_{j}=\theta \beta_{j}+(1-\theta) \omega_{j}$. The combination weight is the linear function of the dynamic adjustment coefficient $\theta$, and the value of $\theta$ is different. The combination weight $\lambda_{j}$ of the parameters also produces linear changes accordingly, as shown in figure 1:

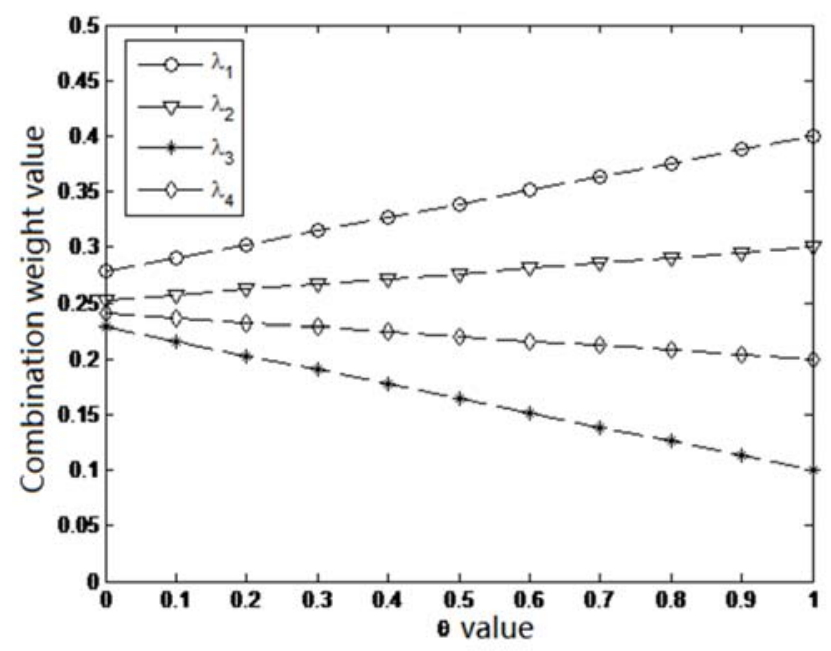

FIGURE 1. The combined weight values under different dynamic adjustment factor $\theta$

Step3: The weighted evaluation of fuzzy soft set $Q$ is calculated.

$\theta$ can be adjusted according to different requirements. Now, the dynamic adjustment coefficient $\theta=0.5$ is taken as an example, and the combined weight is $\lambda_{j}^{0.5}=\{0.3390,0.2763,0.1645,0.2202\}$. The weighted evaluation of fuzzy soft set $Q$, as shown in table 5:

TABLE 5. Weighted evaluation of fuzzy soft set

\begin{tabular}{ccccc}
\hline$(\theta=0.5)$ & $e_{1}$ & $e_{2}$ & $e_{3}$ & $e_{4}$ \\
\hline$x_{1}$ & 0.1356 & 0.2210 & 0.0823 & 0.1101 \\
$x_{2}$ & 0.2373 & 0.1658 & 0.0658 & 0.0881 \\
$x_{3}$ & 0.1695 & 0.1934 & 0.1316 & 0.1101 \\
$x_{4}$ & 0.2034 & 0.1658 & 0.1481 & 0.0881 \\
$x_{5}$ & 0.2712 & 0.0829 & 0.0823 & 0.1762 \\
\hline
\end{tabular}

Step4: Determine the positive ideal solution $v^{+}$and negative ideal solution $v^{-}$for weighted fuzzy soft set $Q$, as shown in table 6: 
TABLE 6. The positive and negative ideal solutions of fuzzy soft set

\begin{tabular}{ccccc}
\hline$(\theta=0.5)$ & $e_{1}$ & $e_{2}$ & $e_{3}$ & $e_{4}$ \\
\hline$v^{+}$ & 0.2712 & 0.2210 & 0.1481 & 0.1762 \\
$v^{-}$ & 0.1356 & 0.0829 & 0.0658 & 0.0881 \\
\hline
\end{tabular}

Step5: To calculate the distance of each decision object to positive and negative ideal solution, the comprehensive evaluation index is obtained, as shown in table 7:

TABLE 7. Comprehensive evaluation index

\begin{tabular}{cccc}
\hline$(\theta=0.5)$ & $x_{i}^{+}$ & $x_{i}^{-}$ & $C_{i}$ \\
\hline$x_{1}$ & 0.1646 & 0.1408 & 0.4610 \\
$x_{2}$ & 0.1369 & 0.1312 & 0.4894 \\
$x_{3}$ & 0.1255 & 0.1348 & 0.5179 \\
$x_{4}$ & 0.1241 & 0.1351 & 0.5212 \\
$x_{5}$ & 0.1530 & 0.1625 & 0.5151 \\
\hline
\end{tabular}

Step6: According to the comprehensive evaluation index, the value of $C_{i}$ is sorted by large to small.

When the dynamic adjustment coefficient is $\theta=0.5$, the decision object of fuzzy soft concentration is sorted and optimized: $x_{4} \succ x_{3} \succ x_{5} \succ x_{2} \succ x_{1}$, thus, object $x_{4}$ is the best fit for the section chief.

When the dynamic regulation coefficient $\theta$ is different, different decision results can be obtained by using the above method, as shown in table 8:

TABLE 8. Dynamic decision results

\begin{tabular}{clc}
\hline$\theta$ & Decision ordering & Decision making \\
\hline 0 & $x_{4} \succ x_{5} \succ x_{1} \succ x_{3} \succ x_{2}$ & $x_{4}$ \\
0.1 & $x_{4} \succ x_{5} \succ x_{1} \succ x_{3} \succ x_{2}$ & $x_{4}$ \\
0.2 & $x_{4} \succ x_{5} \succ x_{1} \succ x_{3} \succ x_{2}$ & $x_{4}$ \\
0.3 & $x_{4} \succ x_{5} \succ x_{1} \succ x_{3} \succ x_{2}$ & $x_{4}$ \\
0.4 & $x_{5} \succ x_{4} \succ\left\{x_{1}, x_{3}\right\} \succ x_{2}$ & $x_{5}$ \\
0.5 & $x_{4} \succ x_{3} \succ x_{5} \succ x_{2} \succ x_{1}$ & $x_{4}$ \\
0.6 & $x_{5} \succ x_{4} \succ x_{2} \succ x_{3} \succ x_{1}$ & $x_{5}$ \\
0.7 & $x_{5} \succ x_{4} \succ x_{2} \succ x_{3} \succ x_{1}$ & $x_{5}$ \\
0.8 & $x_{5} \succ x_{2} \succ x_{4} \succ x_{3} \succ x_{1}$ & $x_{5}$ \\
0.9 & $x_{2} \succ x_{5} \succ x_{4} \succ x_{3} \succ x_{1}$ & $x_{2}$ \\
1 & $x_{2} \succ x_{5} \succ x_{4} \succ x_{3} \succ x_{1}$ & $x_{2}$ \\
\hline
\end{tabular}

Under different dynamic regulation coefficient $\theta$, the optimal decision object is compared, as shown in figure 2: 


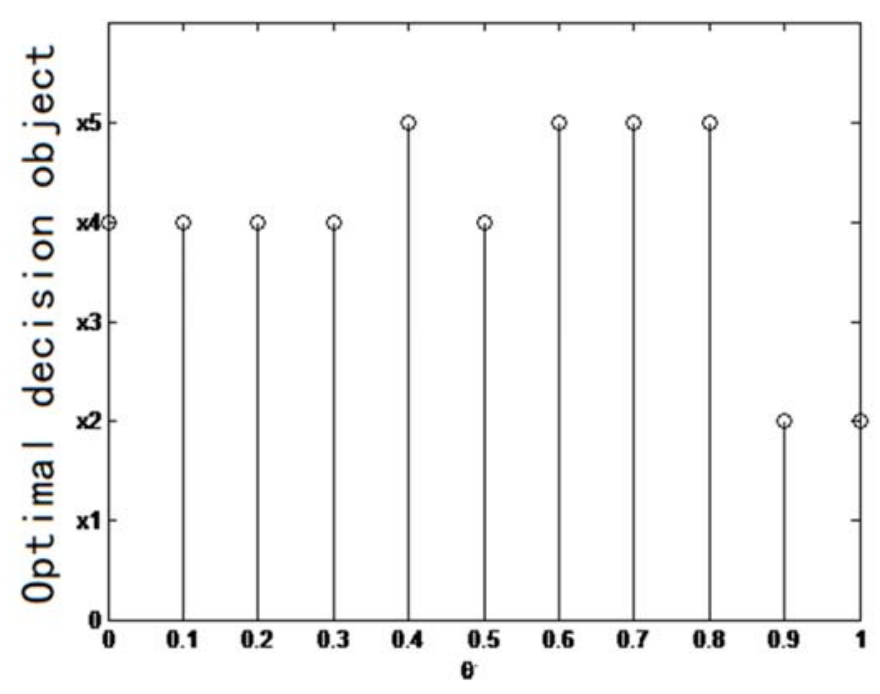

FIGURE 2. Comparison of optimal decision objects

Result analysis:

It can be seen from the decision results that different dynamic adjustment coefficient $\theta$ can get different optimal decision objects. From table 7, you can see that the dynamic adjustment coefficient $\theta=0,0.1$ as optimal search step length of fuzzy soft set decision ordering, get different decisions under dynamic adjustment coefficient $\theta$ sorting and optimal decision objects. When $\theta=0.4$, the occurrence of object $x_{1}$ and object $x_{3}$ is equal, which is because when the TOPSIS method is used to make the decision, the two objects are connected to the positive ideal solution and the negative ideal solution. It can be seen from figure 2 that, in the early stage of dynamic adjustment coefficient, the optimal decision object is $x_{4}$. With the constant change of $\theta$, the optimal decision object is changed from $x_{4}$ to $x_{5}$. When $\theta=0.5$, the optimal decision object is $x_{4}$ and then $x_{5}$ again. When $\theta=0.9$, the optimal decision object is changed from $x_{5}$ to $x_{2}$.This is because the combination weights for the linear function of $\theta$, each property parameters of monotonicity is different, the $\theta$ value is not at the same time, combination weights can affect the decision result, resulting in different optimal decision. On the whole, before $\theta=0.5$, the subjective weight has a great influence on the optimal decision object, and the optimal decision object in the early stage is $x_{4}$. After $\theta=0.5$, the influence of objective weight on the optimal decision object is increasing, and the optimal decision object in the later stage is $x_{5}$. Therefore, it can be obtained that different combination weights can get different optimal decision objects, and the size of weight directly influences the final decision results.

From the perspective of decision-making method, the proposed method can effectively solve the problem that the traditional TOPSIS method must give man-made weight value in decision-making and effectively avoid the subjectivity of decision-making. At the same time, entropy weight method uses the degree of dispersion to determine the objective weight, often ignore the importance of the attribute parameter itself caused by the weight of irrational issues, this paper defines the importance of parameters to improve the entropy method to determine objective weight, Objective weight closer to the actual data, objective weight information more reasonable. By combining the subjective weights and objective weighting decisions through the dynamic adjustment coefficient to form the combined weights, the ratio of the main and objective weights can be dynamically adjusted according to the demand of the decision making so as to achieve the purpose of dynamic decision making and make the decision more flexible and effective. In addition, the method proposed in this paper can be applied to the decision-making of fuzzy soft sets, which can effectively play the role of the parameters of fuzzy soft sets, without the need of data preprocessing in multi-attribute decision making and avoid the decline of decision efficiency due to data preprocessing. Therefore, the decisionmaking method proposed in this paper is more objective, reasonable and effective. 


\section{CONCLUSION}

The research in this paper not only improves the objective weight of the traditional entropy method, but also introduces TOPSIS method into the fuzzy soft set decision-making. It enriches the theory of fuzzy soft set multiattribute decision making, Research has a positive meaning. At the same time, the combination weight proposed in this paper is only about the linear relationship of the dynamic adjustment coefficients. In addition to the linear model, looking for other forms of reference weight with combination weights is the future research direction of this paper.

\section{REFERENCES}

1. Zadeh LA. Fuzzy sets [J]. Information and Control, 1965, 8(3):338-353.

2. Pawlak Z. Rough sets [J]. International Journal of Computer\& Information Sciences, 1982,11(5):341-356.

3. Atanassov K. Intuitionistic fuzzy sets [J]. Fuzzy sets and systems, 1986, 20(1):87-96.

4. Molodtsov D. Soft set theory-first results [J]. Computers and Mathematics with Applicaions, 1999,37(4):19-31.

5. Feng F, Liu X Y, Leoreanu-fotea V, et al. Soft sets and soft Rough Sets[J]. Information sciences, 2011,181(6):1125-1467.

6. Maji P K, Biswas R, Roy A R. Fuzzy soft sets [J]. Journal of Fuzzy Mathematics, 2001, 9:589-602.

7. Maji P K, Roy A R, Biswas R. An application of soft sets in a decision-making problem [J]. Computers and Mathematics with Applications, 2002,44(8-9):1077-1083.

8. Feng F, JunY B, Liu X Y, et al. An adjustable approach to fuzzy soft set based on decision making [J]. Journal of Computational and Applied Mathematics, 2010,234(1):10-20.

9. Zhang Q W, Wang X Q. A New Parameter Reduction Method Based on Soft Set Theory [J]. International Journal of Hybrid Information Technology, 2016, 9(5):99-108.

10. Guan X C, Li Y U,Feng F. A new order relation on fuzzy soft sets and its application [J]. Soft Computer, 2013,17(1):63-70.

11. Zhang Q W, Wang X Q, Zhuang X L. Research of Ranking Method Based on Improved Possible Degree Dominance Relation [J]. Computer Science, 2015, 42(11):274-278.

12. Zhang Q W, Wang X Q. A Multi-Person Decision-making Method Based on Weighted Fuzzy Soft Set[J]. ICIC Express Letters, 2016, 10(1):117-122.

13. Li Z W, Gao N H, Zhang G Q, et al. New methods on parameter reduction of soft sets [J]. Control \& Decision, 2014, 29(7): 1285-1290.

14. Hwang C L, Yoon K. Multiple Attributes Decision Making Methods and Applications [M]. Springer Berlin Heidelberg, 1981.

15. Shan Z P, Zhang Z Z, Ning B Q. Research on Comprehensive Evaluation of Emergency Plan Based on TOPSIS Decision Making Method of Intuitionistic Fuzzy Sets[J]. Mathematics in Practice and Theory 2016, 46(3):160161. 